\title{
Comparative study of wire-integrated urethral catheter versus wire-assisted urethral catheter in difficult male urethral catheterization: a single-center experience
}

\author{
Peng He", Siji Song", Zhipeng Chen, Yang Liu, Zhansong Zhou, Yongquan Wang \\ Department of Urology, Southwest Hospital, Army Medical University, Chongqing 400038, China \\ Contributions: (I) Conception and design: Y Wang, Z Zhou; (II) Administrative support: Y Wang, Z Zhou; (III) Provision of study materials or \\ patients: S Song, Z Chen; (IV) Collection and assembly of data: P He, Y Liu; (V) Data analysis and interpretation: P He, S Song; (VI) Manuscript \\ writing: All authors; (VII) Final approval of manuscript: All authors. \\ "These authors contributed equally to this work. \\ Correspondence to: Yongquan Wang; Zhansong Zhou. Department of Urology, Southwest Hospital, Army Medical University, Chongqing 400038, \\ China. Email: wangyongquan@aliyun.com; zzs68754186@163.com.
}

\begin{abstract}
Background: This study reports the clinical experience of the wire-integrated urethral catheter (WIUC) versus wire-assisted urethral catheter (WAUC) in difficult male urethral catheterization.

Methods: The clinical data of 116 male patients undergoing urinary catheterization with WIUC or WAUC after a failed attempt catheterization in Southwest Hospital from June 2018 to June 2019 were retrospectively analyzed. The time spent during urinary catheterization, cost of consumables, experience rating of catheterization methods and materials assessed by medical staff were compared between WIUC group ( $\mathrm{n}=58)$ and WAUC group $(\mathrm{n}=58)$.

Results: The catheterization succeeded in all 116 patients. The time spent of urinary catheterization was $(4.0 \pm 1.6) \mathrm{min}$ in WIUC group and $(15.4 \pm 3.3) \mathrm{min}$ in WAUC group, and the cost of consumables was $¥ 400$ in WIUC group and $¥ 706.2$ in WAUC group. The medical staff’s experience rating results were $9.0 \pm 0.8$ vs. $7.8 \pm 0.7$ for the catheterization method and $9.3 \pm 0.8$ vs. $8.0 \pm 0.7$ for the materials, respectively, in the WIUC group and WAUC group.
\end{abstract}

Conclusions: WIUC is reasonably designed and easy to operate and can be used for a variety of clinical situations.

Keywords: Wire-integrated urethral catheter; guidewire; male difficult urethral catheterization

Submitted Sep 12, 2019. Accepted for publication Nov 05, 2019.

doi: $10.21037 /$ tau.2019.12.04

View this article at: http://dx.doi.org/10.21037/tau.2019.12.04

\section{Introduction}

Urinary catheterization is widely performed in hospital and is one of the basic clinical skills for medical staff $(1,2)$. However, male catheterization, in particular, can be difficult, especially in patients with enlarged prostate glands or other potentially obstructive conditions in the lower urinary tract, the anxiety of the patient, or unskillful manipulation of medical staff (3), which directly affects the subsequent diagnosis and treatment. Therefore, improving the urinary catheters or the operation techniques is essential for all healthcare personnel to facilitate the procedures of difficult urinary catheterization. At present, the combination use of standard Foley catheter and a guidewire (i.e., wire-assisted urethral catheter, WAUC) is a widely accepted option for difficult catheterization $(4,5)$. On this basis, a wire-integrated urethral catheter (WIUC) (Chongqing Huiquan Technology, China Patent Certificate No.: ZL201420099397.X) was invented and has been used in our center for difficulty catheterization. In this study, we retrospectively compared the effectiveness and safety 

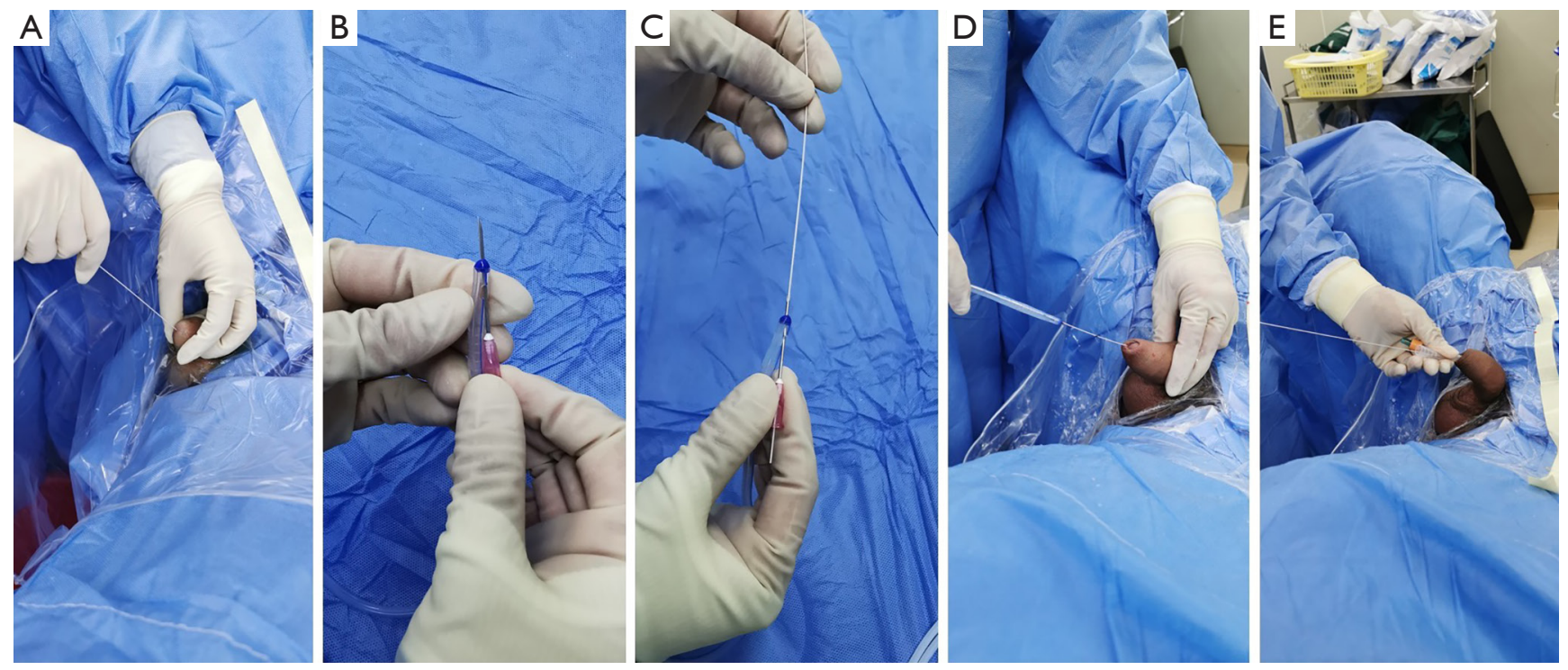

Figure 1 Procedures of wire assisted urethral catheterization. (A) Insert the guidewire into the urethra; (B) puncture on the tip of the catheter with 20G cannula; (C) penetrate the end of the guidewire through the needle; (D) insert the catheter along the guidewire into the bladder; (E) withdraw the guide wire.

of WIUC and WAUC in managing difficult male urethral catheterization.

\section{Methods}

\section{Clinical data}

The clinical data of 116 male patients undergoing urinary catheterization with WIUC or WAUC after a failed attempt of catheterization in our center from June 2018 to June 2019 were retrospectively analyzed. Patients in the WIUC group aged 25-90 years (median age 52 years), among whom 35 patients had benign prostatic hyperplasia, and 8,2 , and 13 patients received the catheterization after transurethral resection of the prostate (TURP), surgery for urethral trauma, or surgery for urethral stricture. Patients in the WAUC group aged 23-88 years (median age 53 years), among whom 41 patients had benign prostatic hyperplasia, and 5,4 , and 8 patients received the catheterization after TURP, surgery for urethral injury, or surgery for urethral stricture.

\section{Study methods}

\section{WAUC}

The patient was in a supine position, and disinfection and draping were routinely performed. The tip of the guidewire
(Cook Medical; Bloomington, 0.035-inch super-slip guide wire) was inserted through the external urethral orifice for about $30 \mathrm{~cm}$. If there was no resistance or kink during the insertion, the guidewire had entered the bladder and was fixed subsequently by assistant. Then, a Foley catheter (Welllead Medical; Guangzhou, 16Fr, FF01B011609) was pierced by a $20 \mathrm{G}$ cannula on the tip and introduced the guidewire into the lumen of the catheter. Then the cannula was withdrawn and the catheter was lubricated with paraffin oil and insert into the bladder along the guidewire. The retention balloon was inflated with sterile water of appropriate volume before the guidewire was removed, and the catheter was connected to a drainage bag (Figure 1).

\section{WIUC}

The patient was in a supine position, and disinfection and draping were routinely performed. The anterior guide wire of the wire-integrated catheter was pulled out for about $30 \mathrm{~cm}$ and inserted through the external urethral orifice. If there was no resistance or kink during the insertion, the guidewire had entered the bladder. After being lubricated with paraffin oil, the catheter was pushed into the bladder along the integrated wire. The retention balloon was inflated with sterile water of appropriate volume. Then, the guidewire was removed, and the catheter was connected to a drainage bag (Figure 2). 

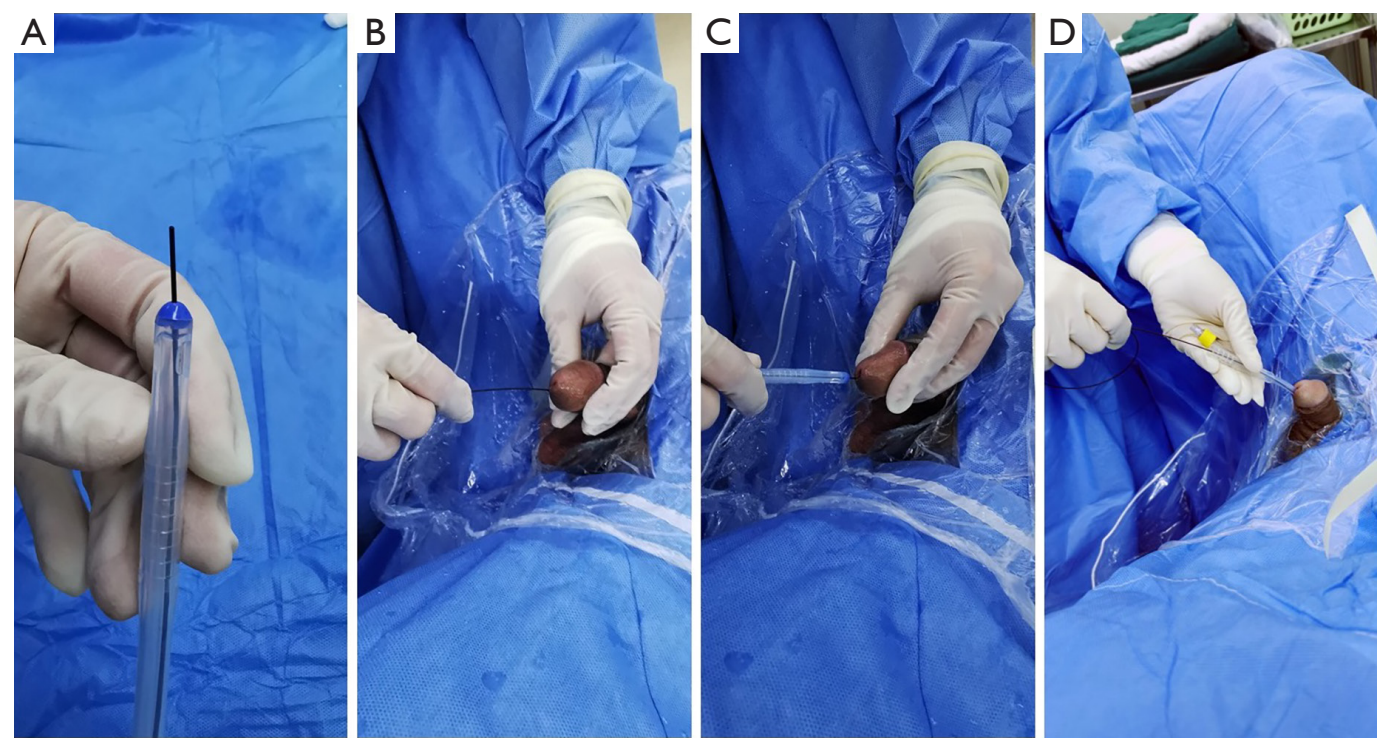

Figure 2 Procedures of wire-integrated urethral catheterization. (A) Front view of wire-integrated urethral catheter; (B) insert the guidewire into the urethra; (C) insert the catheter along the guidewire into the bladder; (D) withdraw the guide wire.

Table 1 Assessment indicators of two catheterization techniques (mean \pm SD)

\begin{tabular}{lccccc}
\hline Group & $\mathrm{n}$ & Operation time $(\mathrm{min})$ & Cost of consumables $(¥)$ & The score of catheterization technique & The score of catheters \\
\hline WAUC group & 58 & $15.4 \pm 3.3$ & 706.2 & $7.8 \pm 0.7$ & $8.0 \pm 0.7$ \\
WIUC group & 58 & $4.0 \pm 1.6$ & 400 & $9.0 \pm 0.8$ & $9.3 \pm 0.8$ \\
$\mathrm{P}$ & $<0.05$ & & $<0.05$ & $<0.05$ \\
\hline
\end{tabular}

WIUC, wire-integrated urethral catheter; WAUC, wire-assisted urethral catheter.

All the operations were performed by seven experienced urologists.

\section{Assessment indicators}

The assessment indicators included the time spent of urinary catheterization, cost of consumables, experience rating of catheterization methods and catheters by medical staff (see Supplementary).

\section{Statistical analysis}

Statistical analysis was performed in SPSS 19.0 software package. The measurement data are expressed by mean $\pm \mathrm{SD}$, and the comparisons between these two groups were performed by $t$-test. $\mathrm{P}$ values $<0.05$ were considered significant.

\section{Results}

The catheterization succeeded in all the 116 patients. The operation time was $(4.0 \pm 1.6) \mathrm{min}$ in WIUC group, which was significantly shorter than that $[(15.4 \pm 3.3) \mathrm{min}]$ in WAUC group $(\mathrm{P}<0.05)$; the cost of consumables was $¥ 400$ in WIUC group, which was significantly lower than that (¥706.2) in WAUC group (Table 1). The medical staff’s scores were $9.0 \pm 0.8 v s$. $7.8 \pm 0.7$ for the catheterization method and $9.3 \pm 0.8$ vs. $8.0 \pm 0.7$ for the consumables, respectively, in the WIUC group and WAUC group (both $\mathrm{P}<0.05$ ). The incidence of complications within 48 hours was $0 \%$ and $3.4 \%$, respectively, in the WIUC group and WAUC group. In one patient of WAUC group, the urinary catheter slipped off, which might be caused by the accidental puncture of the catheter's balloon. A second urinary catheterization was then successfully performed in this patient. 


\section{Discussion}

The adult male urethra is approximately $20 \mathrm{~cm}$ long, with two physiological curvatures and three physiological strictures (6). Any lesion at the urethra may lead to failed urinary catheterization attempt, which make it more difficult for subsequent diagnosis and treatment and also increase patients' suffering. Multiple urinary catheterization techniques and tips have been used for the difficult catheterization (7-9), among which there are three main types of non-instrumental approaches: (I) rigid introducer technique: the metal introducer used as the stent with a diameter slightly smaller than the lumen of the catheter. After bending the leading end of the metal introducer, it was inserted into the catheter as a stent and guided the catheter into the urinary bladder. However, this technique risk urethral trauma and increases the complications and is not routinely recommended. (II) Three hands assistant urinary catheter indwelling: one person inserts the urinary catheter from the external urethral orifice to the prostatic urethra, and the other person lifts the prostatic urethra by exerting pressure inside the anus, to push the urinary catheter forward to pass over the prostatic urethra and make the catheterization possible. The main principle of this method is to lift the urethra to reduce the physiological curvature of the urinary tract, thereby lowering the catheterization resistance. However, this method is feasible only for patients with prostatic hyperplasia, and skillful cooperation between two physicians is needed. (III) Guidewire method (as described above): it takes advantages over the other two methods in the indications (especially for patients experiencing multiple catheterization failures and/or with false passages) and complication controls (false passages and bleeding), and will succeed in $80 \%$ of cases where a urethral catheter has failed (1). However, puncture at the tip of catheter is often tedious, during which the debris of catheter may fall into the bladder (which may cause the bladder stones). Also, there are other conditions such as accidental injury of the fingers of medical staff, difficulty in removing the guidewire due to improper puncturing, catheter balloon damages caused displacement of the catheter, etc. Meanwhile, the use of extra guide wire increased medical expense.

The wire-integrated catheter has been proven safe and effectiveness. As shown in Table 1, WIUC was superior to WAUC in terms of catheterization time, medical expense, and operative convenience. The main advantages of WIUC include saving the time of puncturing and avoid the complications caused by urethral injury and urinary catheter debris. The accidental injury by the needle is also avoided. The operators only need a proper training, and no urological experience is required, which makes it easy to promote. There are some tips for a successful catheterization using WAUC: (I) the successful introduction of the guidewire is the key to success. All parts of the catheter must be fully lubricated. The guidewire must be inserted smoothly. The insertion resistance and refraction must be constantly alert and reinsertion should be performed if that occurs. (II) The guidewire should be inserted for no more than $30 \mathrm{~cm}$, to avoid the guidewire winding in the bladder, which may make it difficult to withdraw. (III) if difficulties occur in removing the guidewire, the catheter should be pulled back and forth together with the guidewire for several times to loosen the winding wire.

Urinary catheterization is a common medical procedure that can lead to significant morbidity and even mortality. Difficult male urethral catheterization should be correctly identified and managed with appropriate techniques and materials (10). WIUC is reasonably designed and easy to operate which can be used for a variety of clinical situations.

\section{Acknowledgments}

Funding: This study was supported by the National Natural Science Foundation of China (No.81400759), and the Chongqing Scientific Foundation (cstc2018jcyjAX0278).

\section{Footnote}

Conflicts of Interest: The authors have no conflicts of interest to declare.

Ethical Statement: The authors are accountable for all aspects of the work in ensuring that questions related to the accuracy or integrity of any part of the work are appropriately investigated and resolved.

\section{References}

1. Sliwinski A, D'Arcy FT, Sultana R, et al. Acute urinary retention and the difficult catheterization: current emergency management. Eur J Emerg Med 2016;23:80-8.

2. Yeh J, Lehrich B, Mesa A, et al. Three-year outcomes of 324 prostate carcinoma patients treated with combination high-dose-rate brachytherapy and intensity modulated 
radiation therapy. Transl Cancer Res 2017;6:269-74.

3. Chen S, Wang P, Dai L, et al. Factors affecting the success rate of first-time urinary catheterization attempt. Chongqing Medicine 2010;39:3449-50.

4. Torricelli FC, De S, Sarkissian C, et al. Hydrophilic guide wires: evaluation and comparison of their properties and safety. Urology 2013;82:1182-6.

5. Jeong SH, Park SJ, Kim YH. Efficacy of urethral catheterisation with a hydrophilic guide wire in patients with urethral trauma for treating acute urinary bladder retention after failed attempt at blind catheterization. Eur Radiol 2012;22:758-64.

6. Yin QQ, Chen LP, Zhang QX, et al. Application of metallic stent catheter in treatment of posterior urethral stricture. Modern Preventive Medicine 2013;40:198-9.

Cite this article as: He P, Song S, Chen Z, Liu Y, Zhou Z, Wang Y. Comparative study of wire-integrated urethral catheter versus wire-assisted urethral catheter in difficult male urethral catheterization: a single-center experience. Transl Androl Urol 2019;8(6):736-740. doi: 10.21037/tau.2019.12.04
7. Liss MA, Leifer S, Sakakine G, et al. The Liss maneuver: a nonendoscopic technique for difficult Foley catheterization. J Endourol 2009;23:1227-30.

8. Kaynar M, Akand M, Goktas S. A novel cannulation technique for difficult urethral catheterization. Arch Ital Urol Androl 2016;88:60-1.

9. Yuminaga Y, Kam J, Louie-Johnsun M, et al. Multi-centre, prospective evaluation of the Seldinger technique for difficult male urethral catheter insertions by non-urology trained doctors. BJU Int 2017;120 Suppl 3:21-7.

10. Davis NF, Quinlan MR, Bhatt NR, et al. Incidence, Cost, Complications and Clinical Outcomes of Iatrogenic Urethral Catheterization Injuries: A Prospective MultiInstitutional Study. J Urol 2016;196:1473-7. 


\section{Supplementary}

Questionnaire on the application of wire-integrated urethral catheter (WIUC) versus wire-assisted urethral catheter (WAUC) in difficult male urethral catheterization

Catheterization method: Wire-integrated urethral catheterization (WIUC) $\square$ Wire-assisted urethral catheter (WAUC) Date:

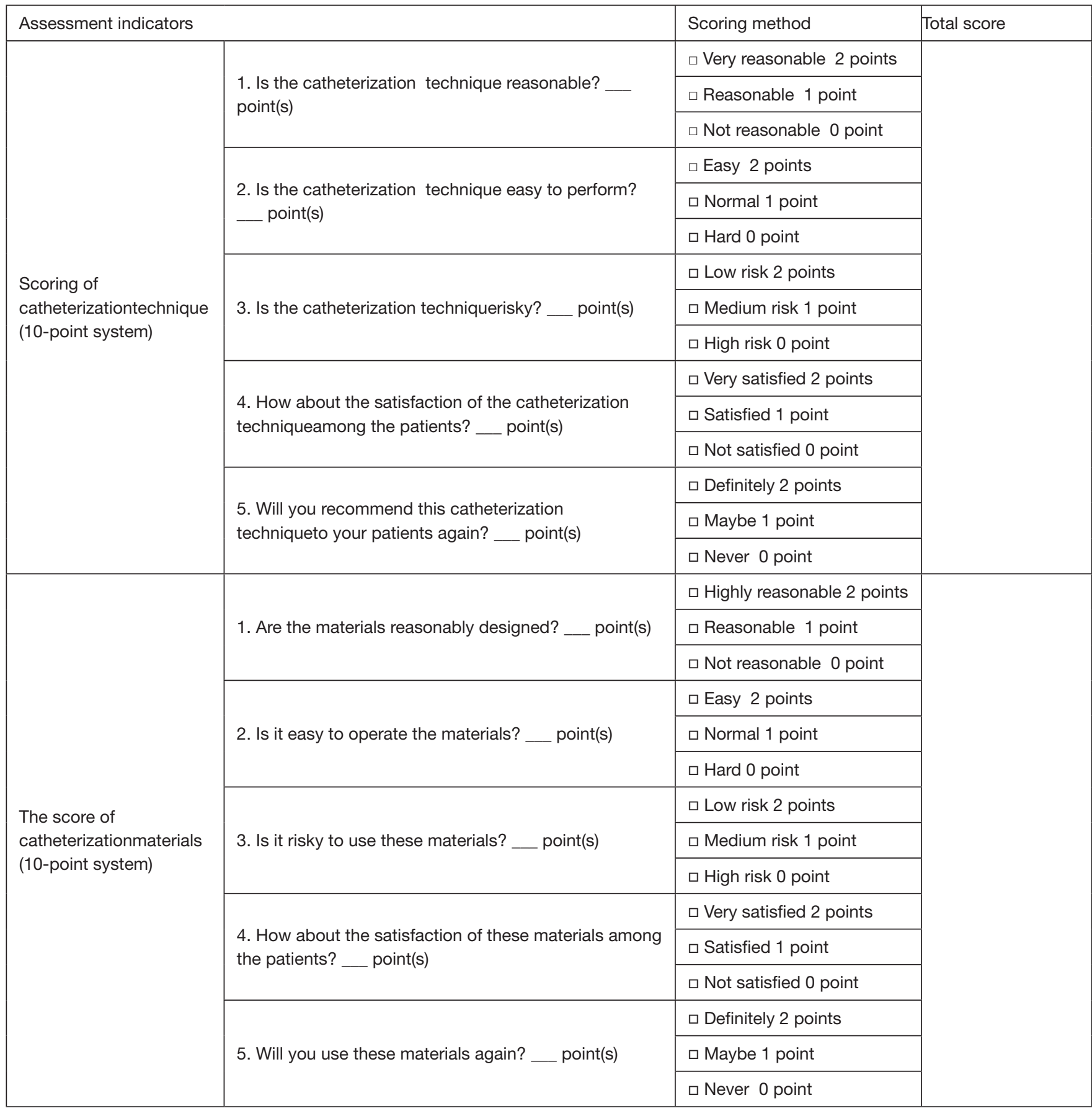

